\title{
Speech Spectrum Modeling for Joint Estimation of Spectral Envelope and Fundamental Frequency
}

\author{
Hirokazu Kameoka, Member, IEEE, Nobutaka Ono, Member, IEEE, and Shigeki Sagayama, Member, IEEE
}

\begin{abstract}
Although considerable effort has been devoted to both fundamental frequency $\left(F_{0}\right)$ and spectral envelope estimation in the field of speech processing, the problem of determining $F_{0}$ and spectral envelopes has largely been tackled independently. If $\boldsymbol{F}_{0}$ were known in advance, then the spectral envelope could be estimated very reliably. On the other hand, if the spectral envelope were known in advance, then we could obtain a reliable $F_{0}$ estimate. $F_{0}$ and the spectral envelope, each of which is a prerequisite of the other, should thus be estimated jointly rather than independently in succession. On this basis, we develop a parametric speech spectrum model that allows us to estimate the $F_{0}$ and spectral envelope simultaneously. We confirmed experimentally the significant advantage of this joint estimation approach for both $F_{0}$ estimation and spectral envelope estimation.
\end{abstract}

Index Terms-Expectation-maximization (EM) algorithm, $F_{0}$ estimation, spectral envelope estimation, speech analysis.

\section{INTRODUCTION}

$\mathbf{S}$ PECTRAL envelope estimation and fundamental frequency $\left(F_{0}\right)$ estimation play very important roles in a wide range of speech processing activities including speech compression, speech recognition, and synthesis. Conventionally, considerable effort has been dedicated to tackling these problems independently. By contrast, this paper describes the importance of estimating the $F_{0}$ and spectral envelope simultaneously. For this purpose, we develop a new speech analyzer based on a parametric speech source-filter model that includes both $F_{0}$ and spectral envelope parameters.

In the filter-based speech synthesis framework, we sometimes assume a speech production model based on a linear system with a single pulse train as the input, which represents a vocal cord excitation. In this model, since the input power spectrum is assumed to have harmonic components with equal powers, a smooth function passing through the prominent spectral peaks corresponds approximately to the power spectrum of the vocal

Manuscript received January 17, 2008; revised September 16, 2008. First published November 17, 2009; current version published July 14, 2010. The associate editor coordinating the review of this manuscript and approving it for publication was Prof. Gaël Richard.

H. Kameoka is with the Media Information Laboratory, NTT Communication Science Laboratories, Kanagawa 243-0198, Japan (e-mail: kameoka@eye.brl. ntt.co.jp).

N. Ono, and S. Sagayama are with Graduate School of Information Science and Technology, The University of Tokyo, Tokyo, 113-8656, Japan (e-mail: onono@ hil.t.u-tokyo.ac.jp; sagayama@ hil.t.u-tokyo.ac.jp).

Color versions of one or more of the figures in this paper are available online at http://ieeexplore.ieee.org.

Digital Object Identifier 10.1109/TASL.2009.2036287 tract impulse response. In this paper, when we refer to "spectral envelope," we assume that it follows this definition.

Many techniques can be used to estimate spectral envelopes. For example, linear predictive coding (LPC) can be understood as a spectral envelope extractor in the sense that it tries to fit an all-pole spectrum to the observed power spectrum using a spectral distortion measure associated with a statistical distribution assumption related to excitation source signals [1]-[5]. For instance, when an excitation source signal is assumed to follow a Gaussian distribution, the corresponding distortion measure is called the Itakura-Saito distance, which imposes larger penalties on negative deviations than on positive deviations of the all-pole spectrum from the observed spectrum. If we can ensure that an excitation source signal follows an assumed distribution, LPC can be a good estimator of a spectral envelope. However, when $F_{0}$ changes, in general the statistics of the excitation signal will change correspondingly. For this reason, we must undertake LPC for each $F_{0}$ with a different assumption regarding the statistical distribution, but if $F_{0}$ is unknown we cannot decide what distribution should be assumed in the first place.

The cepstrum method [6] can also be used to estimate the spectral envelope by low-pass filtering a log-amplitude spectrum interpreted as a signal. But this estimator is also sensitive to $F_{0}$ : the envelope estimate tends to descend into the space between the harmonics.

The discrete all-pole modeling method [7] aims to mitigate this $F_{0}$ dependency problem in LPC, and is designed to fit an all-pole spectrum to a discrete set of harmonic components extracted (via $F_{0}$ estimation) prior to the analysis. A generalized version of the discrete all-pole approach to the estimation of moving average and autoregressive moving average models is proposed in [8]. Similarly, the discrete cepstrum method [9] is an extension of the cepstrum method, and directly estimates the cepstral coefficients where only the extracted harmonics are considered to be the observed data. The regularized discrete cepstrum method [10], [11] applies a regularization technique to the discrete cepstrum approach to impose smoothness conditions on the spectral envelope estimates. STRAIGHT [12], another state-of-the-art technique, begins by estimating $F_{0}$. It then determines an appropriate length for the analysis window according to the $F_{0}$ estimate, and applies discrete Fourier analysis. These methods have been shown to provide high-precision spectral envelope estimation results with a reliable $F_{0}$ estimation preprocessor.

On the other hand, although a huge number of $F_{0}$ estimation algorithms have been developed (see review articles by Hess [13], [14]), their reliability is still limited. One critical issue in $F_{0}$ determination is how to avoid subharmonic or pitch halving 
errors. In a mathematical sense, the period of the signal $s(t)$, which is the inverse of $F_{0}$, is defined as the minimum $T$ value such that $s(t)=s(t+T)$. However, this definition applies strictly only to a perfectly periodic signal and speech departs from perfect periodicity. Therefore, we would like to find the minimum $T$ value such that $s(t) \simeq s(t+T)$. Difficulties include finding a way to measure the degree of periodicity of signals. $T$, which is likely to be the smallest member of an infinite set of time shifts that leave the signal "almost" invariant, cannot be determined easily because if $T$ is the true period, we obtain $s(t) \simeq s(t+n T)$ for all $n \in \mathbb{N}$. We note here that $n T(n \neq 1)$ corresponds to the period of the subharmonic. One effective way to avoid subharmonic errors is to introduce a smoothness measure/constraint for spectral envelopes (that is, limit the variation in partial amplitude across the frequency axis) [15]-[20]. To clarify this point, let us consider a situation where we are given an $F_{0}$ estimate obtained with an $F_{0}$ extractor. If this $F_{0}$ estimate corresponds to $2 T$ (i.e. double the true period), for example, it amounts to assuming that the speech spectrum has a harmonic structure with zero power for all the odd-order harmonics. We may conjecture that the spectral envelope of such a spectrum would be nonsmooth. The smoothness measure therefore signals the irregularity of the speech spectrum when the determined $F_{0}$ value is half the true $F_{0}$.

From the above discussions we can draw the following conclusion: the more reliable the $F_{0}$ determination is the more accurate the spectral envelope estimation becomes, and, on the other hand, the more reliable the spectral envelope estimation is the more reliable the $F_{0}$ determination becomes. Given this chicken-and-egg relationship, $F_{0}$ estimation and spectral envelope estimation should preferably be performed together rather than independently in succession. This is the standpoint we adopt in this paper for formulating a combined model of the spectral envelope and spectral fine structure based on $F_{0}$.

\section{Formulation OF PROPOSED METHOD}

\section{A. Speech Spectrum Modeling}

A short-time segment of a speech signal $y(t)$ can be modeled as an output of the linear system of the vocal tract impulse response $h(t)$ with the source excitation $s(t)$ such that

$$
y(t)=(s(t) * h(t)) w(t)
$$

where $t$ is time and $w(t)$ is a window function. In the Fourier domain, the above equation is written as

$$
Y(\omega)=(S(\omega) H(\omega)) * W(\omega)
$$

where $\omega$ is the frequency, $Y(\omega), S(\omega), H(\omega)$, and $W(\omega)$ are the Fourier transforms of $y(t), s(t), h(t)$, and $w(t)$, respectively. Letting the excitation source signal $s(t)$ be a pulse sequence with a pitch period $T$ such that

$$
s(t)=\sqrt{\frac{T}{2 \pi}} \sum_{n=-\infty}^{\infty} \delta(t-n T)
$$

the Fourier transform of its analytic signal representation is again a pulse sequence given by

$$
\begin{aligned}
S(\omega) & =\sqrt{\frac{T}{2 \pi}}\left[\frac{2 \pi}{T} \sum_{n=0}^{\infty} \delta\left(\omega-n \frac{2 \pi}{T}\right)\right] \\
& =\sqrt{\mu} \sum_{n=0}^{\infty} \delta(\omega-n \mu)
\end{aligned}
$$

where $\mu \equiv 2 \pi / T$ is the $F_{0}$ parameter, $\delta(\cdot)$ the Dirac delta function, and $n$ runs over the integers. Multiplying $S(\omega)$ by the vocal tract frequency response $H(\omega)$ and then taking the convolution with the frequency response $W(\omega)$ of the window function yields the complex spectrum of the short-time segment of voiced speech

$$
\begin{aligned}
Y(\omega) & =(S(\omega) H(\omega)) * W(\omega) \\
& =\sqrt{\mu}\left(\sum_{n=0}^{\infty} H(\omega) \delta(\omega-n \mu)\right) * W(\omega) \\
& =\sqrt{\mu} \sum_{n=0}^{\infty} H(n \mu) W(\omega-n \mu) .
\end{aligned}
$$

We will use the approximation of its power spectrum as a model of the speech spectrum ( Fig. 1)

$$
\begin{aligned}
&|Y(\omega)|^{2}= \mu \sum_{n=0}^{\infty}|H(n \mu)|^{2}|W(\omega-n \mu)|^{2} \\
&+\mu \sum_{n \neq n^{\prime}} H^{*}\left(n^{\prime} \mu\right) H(n \mu) W^{*}\left(\omega-n^{\prime} \mu\right) W(\omega-n \mu) \\
& \simeq \mu \sum_{n=0}^{\infty}|H(n \mu)|^{2}|W(\omega-n \mu)|^{2}
\end{aligned}
$$

This approximation is justified under the sparseness assumption whereby the power spectrum of the sum of multiple signal components is approximately equal to the sum of the power spectra generated independently from the components. The accuracy of this approximation increases as the interferences between the harmonics decrease, where the cross term $W^{*}(\omega-$ $\left.n^{\prime} \mu\right) W(\omega-n \mu)$ is such that $n \neq n^{\prime}$ is sufficiently smaller than $|W(\omega-n \mu)|^{2}$. Assuming $w(t)$ to be a Gaussian window, $|W(\omega)|^{2}$ is also a Gaussian distribution function with a frequency spread of $\sigma$ such that

$$
|W(\omega)|^{2}=\frac{1}{\sqrt{2 \pi} \sigma} \exp \left(-\frac{\omega^{2}}{2 \sigma^{2}}\right)
$$

We see from (6) that the power of each harmonic component is determined according to the spectral envelope function $|H(\omega)|^{2}$. As we want $|H(\omega)|^{2}$ to be a smooth and non-negative function of $\omega$, we introduce the following Gaussian mixture function (see Fig. 2):

$$
|H(\omega)|^{2} \equiv \eta \sum_{m=1}^{M} \frac{\theta_{m}}{\sqrt{2 \pi} \nu_{m}} \exp \left(-\frac{\left(\omega-\rho_{m}\right)^{2}}{2 \nu_{m}^{2}}\right)
$$




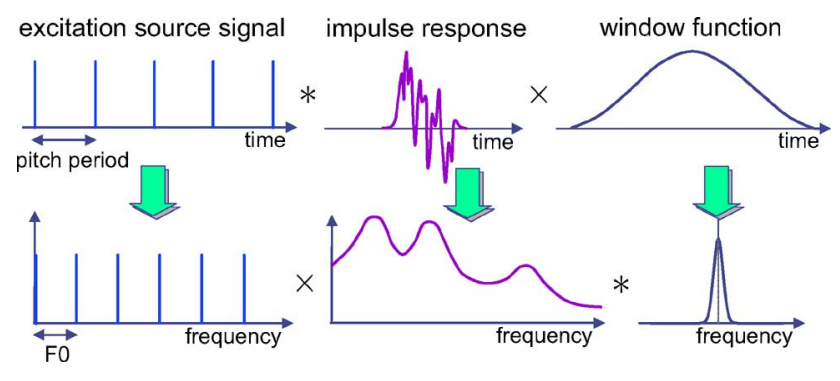

Fig. 1. Linear system approximation model in the power spectrum domain.

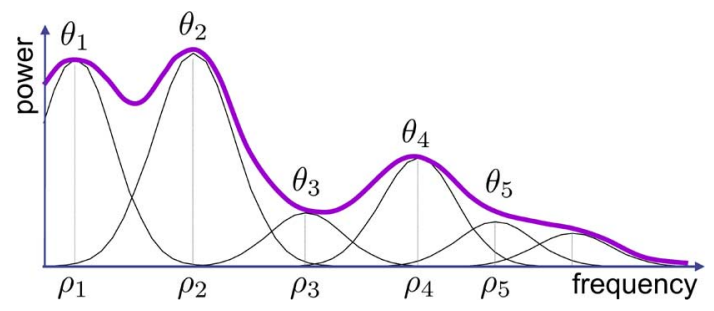

Fig. 2. Spectral envelope model: $|H(\omega)|^{2}$.

with

$$
\sum_{m=1}^{M} \theta_{m}=1
$$

The underlying model for the vocal tract filter with respect to the above spectral envelope model is shown in Appendix I. The scale parameter $\eta$ determines the gain of the spectrum model. From (6) -(8), the speech spectrum can be written concisely as

$$
\begin{aligned}
|Y(\omega)|^{2}= & \mu \sum_{n=0}^{N} \frac{|H(n \mu)|^{2}}{\sqrt{2 \pi} \sigma} \exp \left(-\frac{\left(\omega-n \mu^{2}\right)}{2 \sigma^{2}}\right) \\
= & \sum_{n=0}^{N} \sum_{m=1}^{M} \frac{\eta \mu \theta_{m}}{2 \pi \sigma \nu_{m}} \\
& \times \exp \left(-\frac{(\omega-n \mu)^{2}}{2 \sigma^{2}}-\frac{\left(n \mu-\rho_{m}\right)^{2}}{2 \nu_{m}^{2}}\right)
\end{aligned}
$$

where $n$ is assumed to be bounded for convenience. We notice from (10) that the spectral model we present here is a compound model of two Gaussian mixture models, which represent the spectral envelope and the spectral fine structure, respectively (see Fig. 3). Note that the present model is assumed to contain the null frequency component. Although the null frequency component is usually not considered a partial, this contributes during the model fitting process to preventing the $n=1 \mathrm{com}$ ponent from being pulled into the low-frequency region when a low-frequency noise component is present.

Until now, we have assumed voiced speech with a harmonic structure, but by treating $\sigma$ in (10), which has thus far been constant, as a free parameter, the model can also approximate an unvoiced speech spectrum fairly well. In linear system-based speech synthesis, a white noise signal is often used as the excitation input to synthesize unvoiced speech so the input power

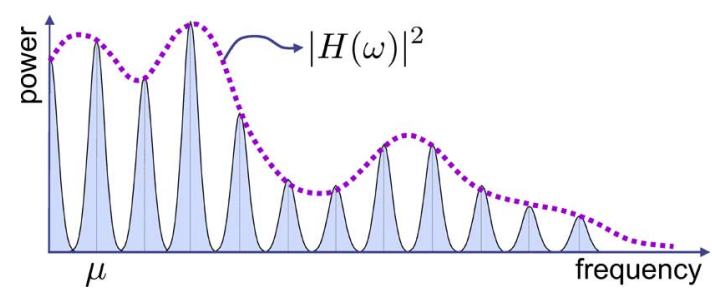

Fig. 3. Compound model of spectral envelope and fine structure $|Y(\omega)|^{2}$.

spectrum should be flat. Now, if $\sigma$ becomes sufficiently large for the tails of adjacent Gaussians to cover each other, the harmonic structure disappears and the model given by (10) appears fairly similar to a white spectrum. However, as the approximation given in (6) becomes less accurate in this case, a more careful modeling of unvoiced speech should be investigated in the future. Another reason for treating $\sigma$ as a free parameter is that it has been effective in avoiding the local minimum problem during the optimization process, which will be described later.

Now, we would like to find the optimal estimates of $\Theta=$ $\left\{\mu, \sigma, \eta, \bigcup_{m=1}^{M}\left\{\rho_{m}, \nu_{m}, \theta_{m}\right\}\right\}$ such that the present model best fits the observed spectrum.

\section{B. Parameter Optimization}

Based on the fact that the present power domain model is composed of multiple Gaussian distribution functions with the mixture weights constrained by each other, the current model fitting problem can be viewed as a natural ${ }^{1}$ extension of Gaussian mixture model estimation using the expectation-maximization (EM) algorithm [21]. This is one advantage we may derive from dealing with the power-domain observation.

Suppose that we are given an observation $F(\omega)$, namely the Fourier transform of a short-term segment of a speech signal. The current problem is to minimize the distortion measure between nonnegative functions $|Y(\omega)|^{2}$ and $|F(\omega)|^{2}$. We define the distortion measure as Csiszár's $I$-divergence [22]

$$
J(\Theta) \equiv \int\left(|F(\omega)|^{2} \log \frac{|F(\omega)|^{2}}{|Y(\omega)|^{2}}-|F(\omega)|^{2}+|Y(\omega)|^{2}\right) \mathrm{d} \omega
$$

which is often used for non-negative matrix factorization (NMF) [23]. It simply reduces to the KL divergence when $\int|F(\omega)|^{2} \mathrm{~d} \omega=\int|Y(\omega)|^{2} \mathrm{~d} \omega=1$. Since the model $|Y(\omega)|^{2}$ contains the parameters that characterize the spectral envelope structure and the spectral fine structure, this optimization leads to a joint estimation of the spectral envelope and $F_{0}$. Note that this measure is not derived from the stochastic modeling of a time domain signal but defined for the sake of convenience when deriving an efficient optimization process.

Now, recall that $|Y(\omega)|^{2}$ is represented in the form

$$
\begin{aligned}
|Y(\omega)|^{2} & =\sum_{n} \sum_{m} \mathcal{Y}_{n, m}(\omega) \\
\mathcal{Y}_{n, m}(\omega) & \equiv \frac{\eta \mu \theta_{m}}{2 \pi \sigma \nu_{m}} \exp \left(-\frac{(\omega-n \mu)^{2}}{2 \sigma^{2}}-\frac{\left(n \mu-\rho_{m}\right)^{2}}{2 \nu_{m}^{2}}\right) .
\end{aligned}
$$

${ }^{1}$ The maximum-likelihood estimation of GMM parameters basically amounts to the problem of fitting a Gaussian mixture density function to a data histogram with the KL divergence as the fitting criterion. 
Based on this fact, we will show that the model parameters can be efficiently estimated with an EM-like iterative algorithm.

First, for any weight functions $\lambda_{n, m}(\omega)$ such that

$$
\forall n, m, \omega: 0<\lambda_{n, m}(\omega)<1
$$

and

$$
\forall \omega: \sum_{n} \sum_{m} \lambda_{n, m}(\omega)=1
$$

we obtain the following inequation:

$$
\begin{aligned}
J(\Theta)=\int\left(|F(\omega)|^{2} \log \frac{|F(\omega)|^{2}}{\sum_{n} \sum_{m} \lambda_{n, m}(\omega) \frac{\mathcal{Y}_{n, m}(\omega)}{\lambda_{n, m}(\omega)}}\right. \\
\left.\quad-|F(\omega)|^{2}+\sum_{n} \sum_{m} \mathcal{Y}_{n, m}(\omega)\right) \mathrm{d} \omega \\
\leq \int\left(|F(\omega)|^{2} \sum_{n} \sum_{m} \lambda_{n, m}(\omega) \log \frac{\lambda_{n, m}(\omega)|F(\omega)|^{2}}{\mathcal{Y}_{n, m}(\omega)}\right. \\
\left.\quad-|F(\omega)|^{2}+\sum_{n} \sum_{m} \mathcal{Y}_{n, m}(\omega)\right) \mathrm{d} \omega
\end{aligned}
$$

by invoking Jensen's inequality based on the concavity of the logarithm function such that

$\log \sum_{i} y_{i} x_{i} \geq \sum_{i} y_{i} \log x_{i}, \Leftrightarrow \log \frac{1}{\sum_{i} y_{i} x_{i}} \leq \sum_{i} y_{i} \log \frac{1}{x_{i}}$

where

$$
\forall i: 0<y_{i}<1, \sum_{i} y_{i}=1
$$

By using $J^{+}(\Theta, \lambda)$ to denote the upper bound of $J(\Theta)$, i.e., the right-hand side of the inequation (15), equality $J^{+}(\Theta, \lambda)=$ $J(\Theta)$ holds if and only if

$$
\forall n, \forall m, \forall \omega: \lambda_{n, m}(\omega)=\frac{\mathcal{Y}_{n, m}(\omega)}{\sum_{n^{\prime}} \sum_{m^{\prime}} \mathcal{Y}_{n^{\prime}, m^{\prime}}(\omega)} .
$$

Equation (17) is obtained by setting the variation of the functional $J^{+}(\Theta, \lambda)$ with respect to $\lambda_{n, m}(\omega)$ at zero. If we are able to decrease $J^{+}(\Theta, \lambda)$ with respect to $\Theta$, then $J(\Theta)$ can be decreased iteratively in the following way.

When $\lambda_{n, m}(\omega)$ is given by (17) with an arbitrary $\Theta$, the objective function $J(\Theta)$ becomes equal to $J^{+}(\Theta, \lambda)$. Then, the parameter $\Theta$ that decreases $J^{+}(\Theta, \lambda)$ while keeping $\lambda_{n, m}(\omega)$ fixed will necessarily decrease $J(\Theta)$, since inequation (15) guarantees that the original objective function is always even smaller than the decreased $J^{+}(\Theta, \lambda)$. Therefore, by repeating the update of $\lambda_{n, m}(\omega)$ by (17) and the update of $\Theta$ that decreases $J^{+}$, the objective function, which is bounded from below by zero, decreases monotonically and converges to a stationary point. This approach is often referred to as the majorization method [24] and has been adopted for various optimization problems including NMF [23].
We notice that $J^{+}$cannot be minimized with respect to $\Theta$ in a closed-form expression because of the third term

$$
\int \sum_{n} \sum_{m} \mathcal{Y}_{n, m}(\omega) \mathrm{d} \omega
$$

More specifically, this part reads

$$
\begin{aligned}
\int \sum_{n} \sum_{m} \mathcal{Y}_{n, m}(\omega) \mathrm{d} \omega \\
=\sum_{n, m} \int \frac{\eta \mu \theta_{m}}{2 \pi \sigma \nu_{m}} \exp \left(-\frac{(\omega-n \mu)^{2}}{2 \sigma^{2}}-\frac{\left(n \mu-\rho_{m}\right)^{2}}{2 \nu_{m}^{2}}\right) \mathrm{d} \omega \\
=\sum_{n, m} \frac{\eta \mu \theta_{m}}{\sqrt{2 \pi} \nu_{m}} \exp \left(-\frac{\left(n \mu-\rho_{m}\right)^{2}}{2 \nu_{m}^{2}}\right)
\end{aligned}
$$

from which we find that $J^{+}(\Theta, \lambda)$ is nonlinear in $\mu, \rho_{m}$ and $\nu_{m}$. However, as this term is the sum of the heights of the sampled points of $|H(\omega)|^{2}$ with an interval $\mu$, it is convenient to approximate it with the integral $\int|H(\omega)|^{2} \mathrm{~d} \omega$. Approximating the Gaussian integral with the Riemann sums with subintervals of an equal length of $\mu$ :

$$
\begin{aligned}
\int \frac{1}{\sqrt{2 \pi} \nu_{m}} \exp & \left(-\frac{\left(\omega-\rho_{m}\right)^{2}}{2 \nu_{m}^{2}}\right) \mathrm{d} \omega \\
& \simeq \mu \sum_{n} \frac{1}{\sqrt{2 \pi} \nu_{m}} \exp \left(-\frac{\left(n \mu-\rho_{m}\right)^{2}}{2 \nu_{m}^{2}}\right)
\end{aligned}
$$

leads us to

$$
\sum_{n} \frac{1}{\sqrt{2 \pi} \nu_{m}} \exp \left(-\frac{\left(n \mu-\rho_{m}\right)^{2}}{2 \nu_{m}^{2}}\right) \simeq \frac{1}{\mu}
$$

since the left-hand side of (20) is 1 . Substituting (21) into (19), it follows that

$$
\int \sum_{n} \sum_{m} \mathcal{Y}_{n, m}(\omega) \mathrm{d} \omega \simeq \sum_{m} \frac{\eta \mu \theta_{m}}{\mu}=\eta .
$$

We can thus conjecture that the third term of the $I$ divergence (and $J^{+}(\Theta, \lambda)$ ) depends very weakly on $\mu, \rho_{m}$, and $\nu_{m}$. The update equations for the parameters other than $\sigma$ and $\eta$ can thus be obtained approximately by maximizing the term

$$
\int|F(\omega)|^{2} \sum_{n} \sum_{m} \lambda_{n, m}(\omega) \log \mathcal{Y}_{n, m}(\omega) \mathrm{d} \omega
$$

Now, the parameter update equations for $\mu, \rho_{m}, \theta_{m}, \sigma, \nu_{m}$, and $\eta$ are obtained as follows (see the Appendix for their derivations):

$$
\begin{aligned}
{\left[\begin{array}{c}
\mu^{(t)} \\
\rho_{1}^{(t)} \\
\vdots \\
\rho_{M}^{(t)}
\end{array}\right]=} & {\left[\begin{array}{cccc}
a & -b_{1} & \cdots & -b_{M} \\
-b_{1} & c_{1} & & 0 \\
\vdots & & \ddots & \\
-b_{M} & 0 & & c_{M}
\end{array}\right]^{-1}\left[\begin{array}{c}
d \\
0 \\
\vdots \\
0
\end{array}\right] } \\
a \equiv & \sum_{n, m} n^{2}\left(\frac{1}{\sigma^{(t-1) 2}}+\frac{1}{\nu_{m}^{(t-1)_{2}}}\right) \\
& \times \int \lambda_{n, m}(\omega)|F(\omega)|^{2} \mathrm{~d} \omega
\end{aligned}
$$



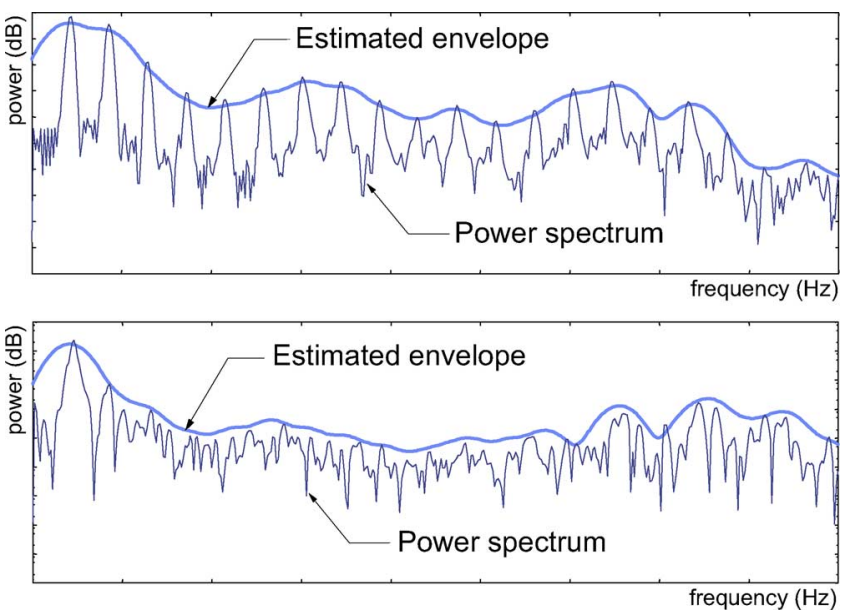

Fig. 4. Observed power spectra of voiced (top) and unvoiced (bottom) speech and the corresponding spectral envelope estimates.

$$
\begin{aligned}
b_{m} & \equiv \frac{1}{\nu_{m}^{(t-1)} 2} \sum_{n} n \int \lambda_{n, m}(\omega)|F(\omega)|^{2} \mathrm{~d} \omega \\
c_{m} & \equiv \frac{1}{\nu_{m}^{(t-1)} 2} \sum_{n} \int \lambda_{n, m}(\omega)|F(\omega)|^{2} \mathrm{~d} \omega \\
d & \equiv \frac{1}{\sigma^{(t-1) 2}} \sum_{n} n \sum_{m=1}^{M} \int \lambda_{n, m}(\omega)|F(\omega)|^{2} \omega \mathrm{d} \omega \\
\theta_{m}^{(t)}= & \frac{1}{F} \sum_{n} \int \lambda_{n, m}(\omega)|F(\omega)|^{2} \mathrm{~d} \omega \\
\sigma^{(t) 2}= & \frac{1}{F} \sum_{n, m} \int \lambda_{n, m}(\omega)|F(\omega)|^{2} \\
\nu_{m}^{(t) 2}= & \left.\frac{1}{F} \sum_{n}\left(n \mu^{(t)}-\rho_{m}^{(t)}\right)^{2}\right)^{2} \mathrm{~d} \omega \\
& \times \int \lambda_{n, m}(\omega)|F(\omega)|^{2} \mathrm{~d} \omega \\
\eta^{(t)}= & F / \sum_{n, m} \frac{\mu^{(t)} \theta_{m}^{(t)}}{\sqrt{2 \pi} \nu_{m}^{(t)}} \\
& \times \exp \left(-\frac{\left(n \mu^{(t)}-\rho_{m}^{(t)}\right)^{2}}{2 \nu_{m}^{(t)} 2}\right)
\end{aligned}
$$

where $F=\int|F(\omega)|^{2} \mathrm{~d} \omega$ and the superscript $t$ refers to the iteration cycle. Some examples of the estimated envelope $|H(\omega)|^{2}$ with $M=15$ can be seen in Fig. 4.

\begin{tabular}{|c|c|c|c|}
\hline \multirow{2}{*}{ Speech File } & \multicolumn{2}{|c|}{$F_{0}$ accuracy $(\%)$} & \multirow{2}{*}{ Cosine $(\%)$} \\
\hline & $\pm 5 \%$ & $\pm 20 \%$ & \\
\hline 1 & $98.4(85.3)$ & $98.6(98.6)$ & 96.7 \\
\hline myis $\overline{\text { da }} \overline{0} 2^{-}$ & $\overline{93 . \overline{3}}(\overline{82} . \overline{6})$ & $\overline{97 . \overline{8}}(\overline{97 . \overline{8}}$ & $9 \overline{8.0}$ \\
\hline myiso & $\overline{94} . \overline{2}(\overline{79} . \overline{9}$ & $\overline{97 . \overline{5}}(\overline{96} \overline{9}$ & $9 \overline{6} .0$ \\
\hline myiso & $\overline{98} . \overline{0}(\overline{8} 6 . \overline{3})$ & $\overline{99} . \overline{0}(\overline{95} . \overline{1} \overline{)}$ & $\overline{9} \overline{6.8}$ \\
\hline myisd & $\overline{93} . \overline{7}(\overline{7} 1 . \overline{7})$ & $\overline{97 . \overline{8}} \overline{(96 . \overline{1}) \bar{s}}$ & $9 \overline{5.9}$ \\
\hline fyms da $0 \overline{1}$ & $\overline{97 . \overline{2}}(\overline{87 . \overline{0}})$ & $\overline{98} . \overline{0}(\overline{98} . \overline{0}) \bar{c}$ & $9 \overline{8.3}$ \\
\hline fyms da $\overline{0} 2$ & $\overline{96 . \overline{8}}(\overline{8} 8 . \overline{5})$ & $\overline{98} . \overline{1} \overline{(98 . \overline{1}}$ & $9 \overline{7} .6$ \\
\hline 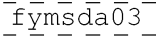 & $\overline{95 . \overline{4}}(\overline{8} 4 . \overline{6})$ & $\overline{98.5}) \overline{9} \overline{9}$. & $9 \overline{8.2}$ \\
\hline fyms da & $\overline{97 . \overline{0}}(\overline{8} 8 . \overline{2})$ & $\overline{98} . \overline{1}(\overline{98} . \overline{1})$ & $9 \overline{8.2} 2^{-}$ \\
\hline fyms da $0 \overline{0} 5^{-}$ & $\overline{95 . \overline{7}}(\overline{8} 6 . \overline{5})$ & $\overline{99} . \overline{2}(\overline{98} \overline{.5})$ & $9 \overline{8.1}$ \\
\hline
\end{tabular}

\section{EXPERIMENTAL EVALUATIONS}

\section{A. Single Voice $F_{0}$ Estimation}

To confirm its performance as an $F_{0}$ extractor, we tested our method on ten Japanese speech data of male ("myi") and female ("fym") speakers from the ATR speech database [25] and chose the well-known $F_{0}$ extractor "YIN" [26] for comparison. All the speech data were around $5 \mathrm{~s}$ long, monaural, and sampled
TABLE I

ACCURACIES OF $F_{0}$ ESTIMATION

at $16 \mathrm{kHz}$. All the power spectra were computed using a Hanning window that was $32 \mathrm{~ms}$ long with a 10-ms overlap. The spectral model was made using $N+1=60$ Gaussians, and the envelope model was made using $M=15$ Gaussians. The number of free parameters was therefore $3+15 \times 3=48$. Three independent sets of iterations were run for each analysis frame and the starting conditions were different in each case. The initial values of $\mu$ were set at 47,94 , and $141 \mathrm{~Hz}$, respectively, and of these conditions, the converged parameter set that gave the minimum $J$ value was considered to be the global optimum. As the present optimization algorithm usually converged after ten iterations, each search was run for ten iterations. The initial $\theta_{m}$ values were determined uniformly, and $\sigma$ and $\nu_{m}$ were initialized at 31 and $313 \mathrm{~Hz}$, respectively. $\rho_{m}$ was initially set at 8000/m Hz.

For an $F_{0}$ estimation task, we defined two error criteria, namely deviations over $5 \%$ and $20 \%$, from the hand-labeled $F_{0}$ reference as fine and gross errors, respectively. The former criterion shows how precisely the proposed analyzer is able to estimate $F_{0}$ and the latter reveals the robustness with respect to pitch doubling/halving pitch errors. The areas where reference $F_{0}$ s were given by zero were not considered in the accuracy computation. As a second evaluation, we took the average of the cosine measures between $|Y(\omega)|^{2}$ and $|F(\omega)|^{2}$ for the entire analysis interval to confirm the appropriateness of the choices of distortion measure for minimization and of the model for expressing speech power spectra. These results can be seen in Table I. The numbers in the brackets in Table I are the results obtained with YIN. The source code was kindly provided to us by its authors. The results confirm that our method is as accurate as YIN when it comes to roughly estimating $F_{0}$, and significantly outperforms YIN as regards precise estimation. Thus, our method would be especially useful when a very precise $F_{0}$ estimate is required, which is exactly the case with spectral envelope estimation algorithms that use $F_{0}$ estimates. We should note however that the parameters used for YIN may not do it full justice. The results seem to be rather good for a frame-by-frame algorithm, which encourages us to embed this envelope structured model into the parametric spectrogram model proposed in [27], [28] to exploit the temporal connectivity of speech attributes and thus realize a further improvement.

Although we have chosen $N$ and $M$ (the number of Gaussian components for the spectral envelope model and the spectral 
fine structure model) experimentally, we infer that the performance as an $F_{0}$ extractor would depend strongly on the model order. Determining the model order remains an open problem that must be investigated.

\section{B. Synthesis and Analysis}

Here we evaluate the accuracy of spectral envelope estimation. To accomplish this, we must use speech signals as the experimental data whose true spectral envelope is known in advance. For this purpose, we created several synthetic speech signals, which were made using three types of linear filter, namely an all-zero filter, an all-pole filter and a pole-zero filter, and the input excitation. The input excitation we used here is a linear chirped single pulse signal, whose $F_{0}$ modulates linearly from 100 to $400 \mathrm{~Hz}$ within 2 seconds. We chose filters with the following characteristics:

$$
\begin{aligned}
\mathcal{H}(z)=\prod_{i=1}^{8}\left(1-\beta_{i} z^{-1}\right) / \prod_{j=1}^{5}\left(1-\alpha_{j} z^{-1}\right) . \\
\text { All-zero }(1): \beta_{1}=\beta_{2}^{*}=0.7919+0.5365 \mathrm{j} \\
\beta_{3}=\beta_{4}^{*}=0.3744+0.8397 \mathrm{j} \\
\beta_{5}=\beta_{6}^{*}=-0.8290+0.3063 \mathrm{j} \\
\beta_{7}=\beta_{8}^{*}=-0.2873+0.8632 \mathrm{j} \\
\alpha_{1}=\ldots=\alpha_{5}=0 \\
\beta_{2}=-0.8365 \\
\beta_{3}=\beta_{4}^{*}=0.7032+0.7269 \mathrm{j} \\
\beta_{5}=\beta_{6}^{*}=-0.0347+1.0159 \mathrm{j} \\
\beta_{7}=\beta_{8}^{*}=-0.7923+0.5840 \mathrm{j} \\
\alpha_{1}=\cdots=\alpha_{5}=0 \\
\beta_{1}=\ldots=\beta_{8}=0 \\
\alpha_{1}=\alpha_{2}^{*}=-0.5026+0.5976 \mathrm{j} \\
\alpha_{3}=\alpha_{4}^{*}=0.4225+0.7529 \mathrm{j} \\
\alpha_{5}=0.6602
\end{aligned}
$$

$$
\text { Pole - zero : } \beta_{1}=1.1675
$$

$$
\begin{aligned}
& \beta_{2}=-0.8365 \\
& \beta_{3}=\beta_{4}^{*}=0.7032+0.7269 \mathrm{j} \\
& \beta_{5}=\beta_{6}^{*}=-0.0347+1.0159 \mathrm{j} \\
& \beta_{7}=\beta_{8}^{*}=-0.7923+0.5840 \mathrm{j} \\
& \alpha_{1}=\alpha_{2}^{*}=-0.5026+0.5976 \mathrm{j} \\
& \alpha_{3}=\alpha_{4}^{*}=0.4225+0.7529 \mathrm{j} \\
& \alpha_{5}=0.6602 .
\end{aligned}
$$

The frequency responses of the respective filters can be seen in Fig. 5. As a measure for assessing the accuracy of the spectral envelope estimation we chose the "Spectral Distortion (SD)," defined by

$$
\frac{1}{I} \sum_{i=1}^{I}\left(\log \left|H\left(\omega_{i}\right)\right|-\log \left|\mathcal{H}\left(e^{j \omega_{i}}\right)\right|\right)^{2}
$$

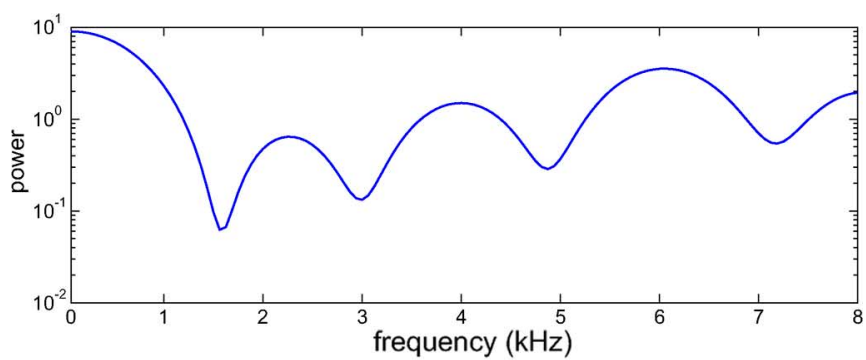

(a)

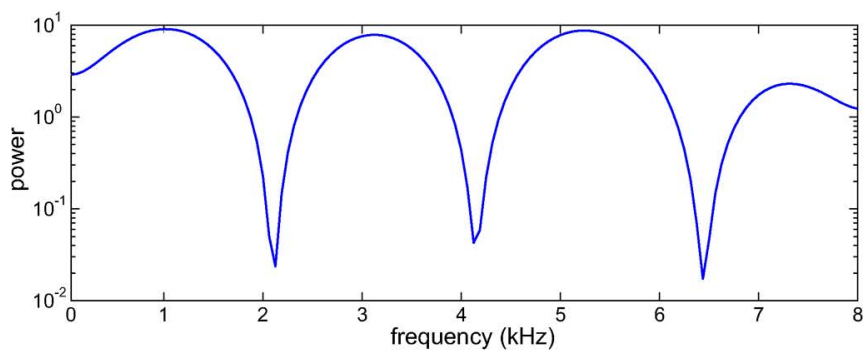

(b)

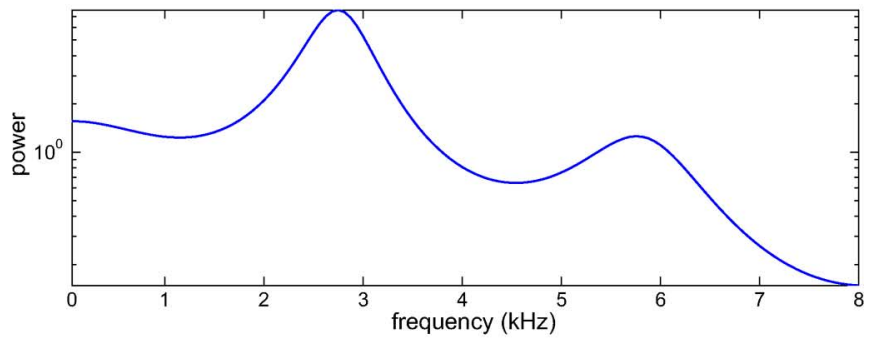

(c)

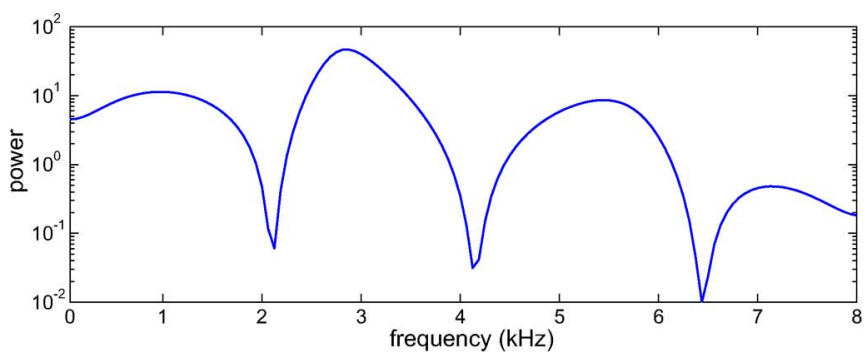

(d)

Fig. 5. Frequency responses of the synthesis filters. (a) All-zero type (1). (b) All-zero type (2). (c) All-pole type. (d) Pole-zero type.

where $i$ refers to the frequency-bin index, $\left|\mathcal{H}\left(e^{j \omega_{i}}\right)\right|$ is the true (reference) spectral envelope, and $\left|H\left(\omega_{i}\right)\right|$ is the spectral envelope estimate.

The experimental results are shown in Fig. 6. Fig. 6(a)-(d) are the results when the tests were undertaken using the data created respectively by all-zero (1), all-zero (2), all-pole, and pole-zero. Each graph shows the transitions of SD values within two seconds during which the $F_{0}$ of the input excitation modulates from 100 to $400 \mathrm{~Hz}$. These graphs show that as the $F_{0}$ of the input increases, conventional methods such as 40-order LPC and LPC cepstrum tend to obtain poorer results. This is perhaps because the envelope estimates descend into the space between the partials for high $F_{0}$ values. The accuracy of the envelope estimates obtained with the proposed method does not seem to deteriorate even with high $F_{0}$ values. This is obviously because the proposed method attempts to estimate the spectral 


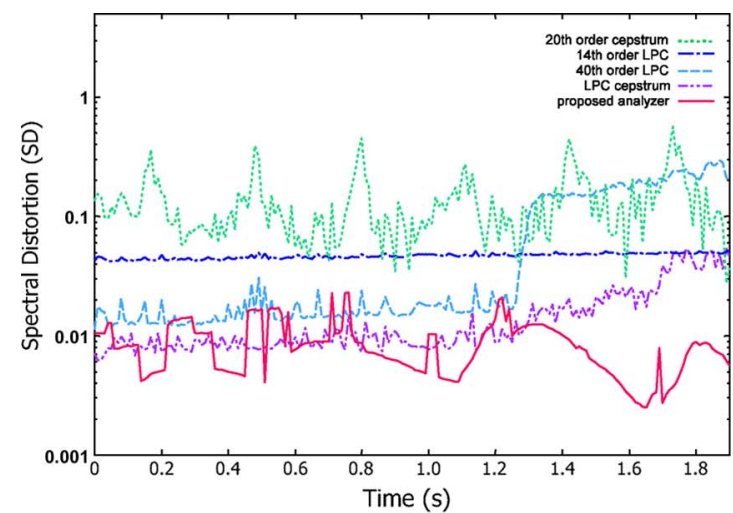

(a)

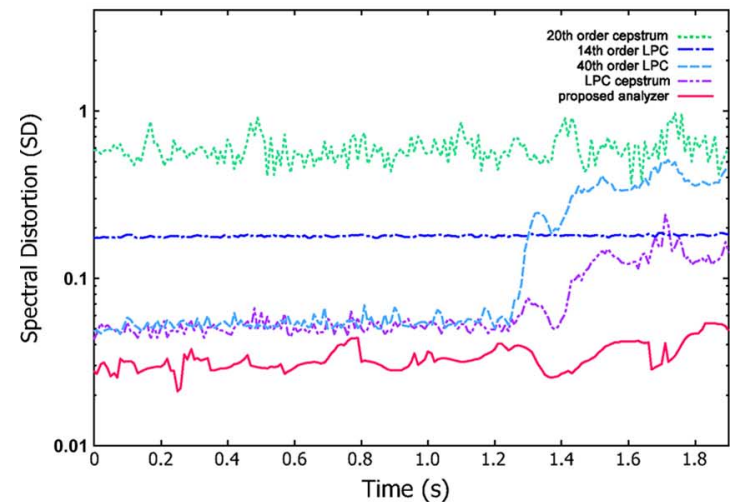

(b)

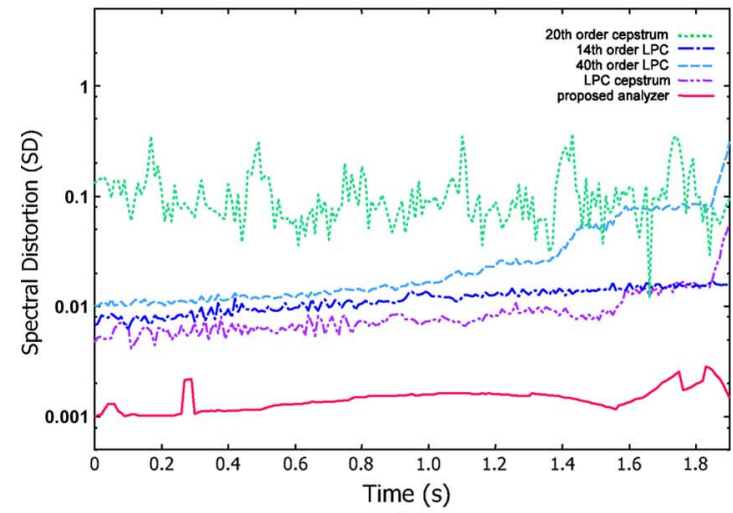

(c)

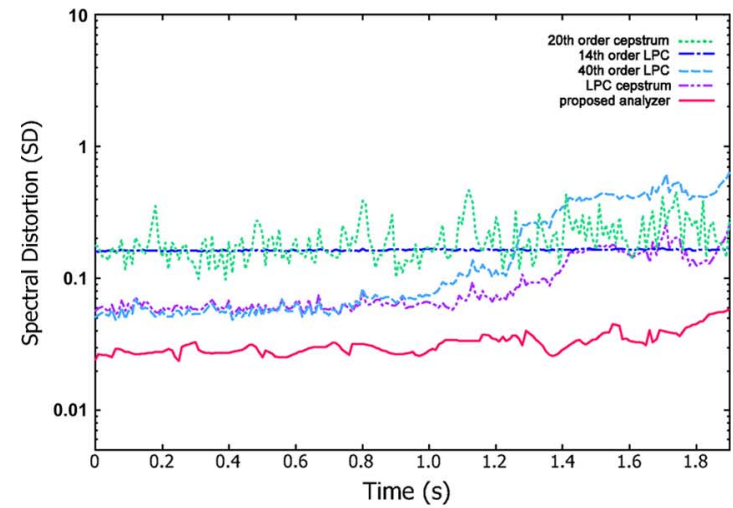

(d)

Fig. 6. Comparison of the accuracies of spectral envelope estimation with the proposed method and the conventional methods. Each graph shows the transitions of SD values during two seconds. (a) All-zero type (1). (b) All-zero type (2). (c) All-pole type. (d) Pole-zero type.

fine structure at the same time. On the other hand, the 14-order
TABLE II

PREFERENCE SCORE (\%) OF THE SyNTHESIZEd SPEECH GENERATEd BY THE PROCEDURE DESCRIBED IN SECTION III-C

\begin{tabular}{|c|c|c|}
\hline istener & vowel & word \\
\hline$\stackrel{\mathrm{A}}{-}$ & 60 & 8 \\
\hline & $-\overline{60}$ & 83 \\
\hline & $-\overline{40}$ & 68 \\
\hline & $-8 \overline{80}-$ & 80 \\
\hline & $-\overline{60}$ & 95 \\
\hline & 80 & 96 \\
\hline & $1 \overline{0} 0^{-}$ & $10 \overline{0}$ \\
\hline & $-\overline{40}$ & $64^{-}$ \\
\hline & $8 \overline{80}$ & 94 \\
\hline $\bar{J}$ & 60 & 88 \\
\hline Ave. & 66 & 83 \\
\hline
\end{tabular}

LPC envelope is too smooth to realize a good fit with the true envelope, and the cepstrum method always seems to obtain poorer results.

\section{Analysis and Synthesis}

As the advantage of the filter-type speech synthesis framework is its flexibility as regards modifying speech, here we evaluate the basic performance of the present method as a speech modifier.

We performed a psychological experiment to evaluate the intelligibility of pitch-modified synthesized speech generated by using the parameters obtained with the present and LPC analyzers. The parameters extracted using the present analyzer were transformed into a synthesized speech signal using the time-domain representation of the present model described in Appendix I. As we see from (30), a vocal tract impulse response $h(t)$ can be constructed employing the estimates of $\theta_{m}, \nu_{m}$, and $\rho_{m}$, and using randomly chosen $\phi_{m}$. Then, we can simply generate a synthesized signal by convolving a pulse train (with an interval of a certain period) with the constructed $h(t)$. As the test set, we used speech data consisting of five vowels $(/ \mathrm{a} /, \mathrm{i} /$, $/ \mathrm{u} /, / \mathrm{e} /, / \mathrm{o} /$ ) and 40 randomly chosen words uttered by a female speaker that were excerpted from the same database. Analyses were performed using a Hanning window that was $32 \mathrm{~ms}$ long with a 10-ms overlap. The dimension of the parameters for the proposed model was set at 45 . In this experiment, the LPC order was set at 45 so that the number of degrees of freedom would be the same in both models, thus allowing for a fairer comparison. For the LPC analysis, the $F_{0}$ s were extracted by using the supplementary $F_{0}$ extraction tool included in the Snack Sound Toolkit. ${ }^{2}$ Each synthesized speech used for the evaluation was excited with an estimated vocal tract characteristic by a pulse sequence at intervals with a different pitch period from the original one. The pitch periods were modified to $80 \%$ and $120 \%$ of the pitch periods obtained from the original speech. We let ten listeners choose the speech that they thought was the more intelligible and obtained a preference score for the results using the proposed analyzer. The preference score, shown in Table II, shows that the intelligibility of the synthesized speech generated by the present analyzer is higher than that generated by the LPC analyzer.

${ }^{2}$ [Online]. Available: http://www.speech.kth.se/snack/ 
It should be noted that as LPC models of order 12 or 14 are often considered to be appropriate for the synthesis of speech vowels, different settings for the LPC model may have yielded better performance.

\section{CONCLUSION}

In this paper, we formulated $F_{0}$ determination and spectral envelope estimation as a joint optimization problem with respect to a composite function model of the spectral envelope and the spectral fine structure. The experiments confirmed the effectiveness of our method as an $F_{0}$ extractor, spectral envelope extractor, and speech modifier.

The extension of the present model to concurrent utterances of multiple speakers is straightforward. We can construct a mixed speech spectrum model by mixing the speech spectrum models introduced in this paper each of which has its own degree of freedom. The derivation of the optimization algorithm is exactly the same as the derivation described in this paper. In future, we will examine its application to monaural speech separation.

\section{APPENDIX I}

\section{DERIVATION OF SPECTRAL ENVELOPE MODEL}

Here we show the underlying model for the vocal tract filter that leads to the spectral envelope model introduced in Section II-A.

Because human vocal tracts are at most about $20 \mathrm{~cm}$ long, it would be fairly natural to assume that vocal tract impulse responses are dominant around $t=0$. Based on this assumption, we consider modeling a vocal tract impulse response by superimposing several Gabor functions, all of which are centered at $t=0$. Using an analytic signal representation, we define the impulse response $h(t)$ as

$$
h(t)=\sum_{m=1}^{M} \sqrt{\theta_{m}} \exp \left(-\nu_{m}^{2} t^{2}\right) \exp \left(-\mathrm{j} \rho_{m} t\right) e^{\mathrm{j} \phi_{m}}
$$

where $\rho_{m}, \nu_{m}, \theta_{m}$, and $\phi_{m}$ represent the carrier frequency, amplitude modulation parameter, scale, and starting phase for the carrier sinusoid of the $m$ th Gabor function, respectively. It should be noted that in a strict sense this model is not valid as regards allowing the filter to be acausal. Now, the Fourier transform of $h(t)$ (see Fig. 7) is written as

$$
H(\omega)=\sum_{m} \frac{\sqrt{\theta_{m}}}{\sqrt{2 \nu_{m}^{2}}} \exp \left(-\frac{\left(\omega-\rho_{m}\right)^{2}}{4 \nu_{m}^{2}}\right) e^{\mathrm{j} \phi_{m}} .
$$

The power spectrum is then given as

$$
\begin{aligned}
& |H(\omega)|^{2}=\sum_{m} \frac{\theta_{m}}{2 \nu_{m}^{2}} \exp \left(-\frac{\left(\omega-\rho_{m}\right)^{2}}{2 \nu_{m}^{2}}\right) \\
& \quad+\sum_{k \neq l} \frac{\sqrt{\theta_{k} \theta_{l}}}{2 \nu_{k} \nu_{l}} \exp \left(-\frac{\left(\omega-\rho_{k}\right)^{2}}{4 \nu_{k}^{2}}-\frac{\left(\omega-\rho_{l}\right)^{2}}{4 \nu_{l}^{2}}\right) e^{\mathrm{j}\left(\phi_{k}-\phi_{l}\right)} .
\end{aligned}
$$

Assuming that $\phi_{m}$ is an independent random variable uniformly distributed on the interval $D=[-\pi, \pi)$, the second term can be canceled out by taking the expectation of $|H(\omega)|^{2}$ with respect to $\phi_{m}$ such that

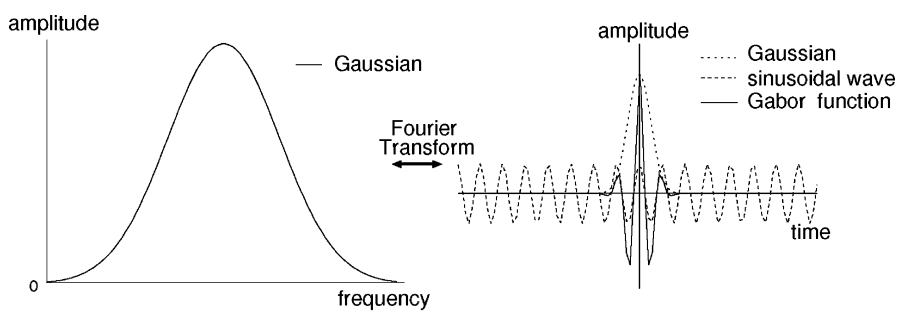

(a)

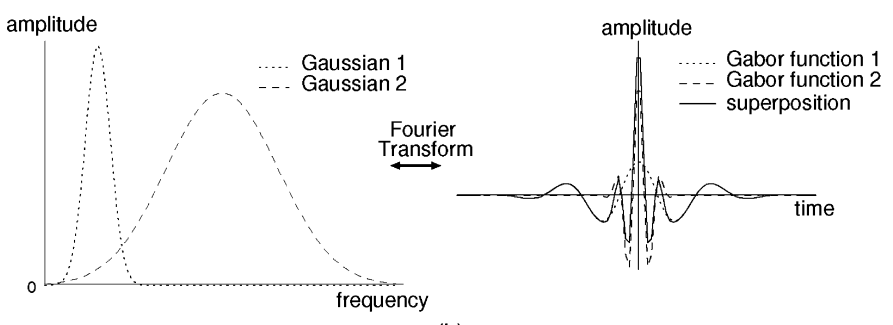

(b)

Fig. 7. Equations (30) and (31) with $\phi_{m}=0$ for (a) $M=1$ and (b) $M=2$. (a) Single Gaussian case. (b) Gaussian mixture case.

$$
\mathrm{E}\left\{|H(\omega)|^{2}\right\}=\sum_{m} \frac{\theta_{m}}{2 \nu_{m}^{2}} \exp \left(-\frac{\left(\omega-\rho_{m}\right)^{2}}{2 \nu_{m}^{2}}\right) .
$$

We shall assume that $|H(\omega)|^{2} \simeq \mathbb{E}\left\{|H(\omega)|^{2}\right\}$ and use (32) to express spectral envelopes. By imposing a scale constraint

$$
\int|H(\omega)|^{2}=\eta, \quad \sum_{m} \theta_{m}=1
$$

we finally arrive at (8).

We notice from an inspection of (30) and (32) that constraining each Gabor function in (30) so that they are localized densely around $t=0$, which can be accomplished simply by setting $\nu_{m}$ at a large value, amounts to imposing a smoothness constraint on the spectral envelope, as each Gaussian function in (32) spreads in proportion to $\nu_{m}$.

\section{APPENDIX II}

\section{DERIVATION OF UPDATE EQUATIONS}

Update equations for $\theta$ guaranteeing approximately the nonincrease of $J^{+}$can be derived in various ways, only two of which are described in this section owing to space limitations.

One way involves adopting a pure coordinate descent approach. That is, we seek to find the minimum of $J^{+}$with respect to each parameter while keeping the other parameters fixed at the newest update values. As for $\mu, \rho_{m}, \theta_{m}$, and $\nu_{m}$, the update equations can be achieved approximately by maximizing (23). Taking the partial derivative of (23) with respect to each of the parameters and setting at zero, we obtain the following:

$$
\begin{aligned}
& \mu^{(t)}=\frac{g+\sqrt{g^{2}+4 a F}}{2 a}, \quad \rho_{m}^{(t)}=\frac{b_{m}}{c_{m}} \mu^{(t)}, \\
& \theta^{(t)}=\text { Eq. }(25), \quad \nu_{m}^{(t)}=\text { Eq. }(27)
\end{aligned}
$$

where

$$
g \equiv d+\sum_{m} \rho_{m}^{(t-1)} b_{m}
$$


As for $\sigma$ and $\eta, J^{+}$can be directly minimized with (26) and (28).

The second way is to adopt an approximation technique. This approach also adopts the coordinate descent approach but differs in that we further approximate (23) with

$$
\begin{aligned}
\int|F(\omega)|^{2} \sum_{n, m} \lambda_{n, m}(\omega) & \left(\log \frac{\eta \mu^{(t-1)} \theta_{m}}{2 \pi \sigma \nu_{m}}\right. \\
- & \left.\frac{(\omega-n \mu)^{2}}{2 \sigma^{2}}-\frac{\left(n \mu-\rho_{m}\right)^{2}}{2 \nu_{m}^{2}}\right) \mathrm{d} \omega
\end{aligned}
$$

which can be minimized jointly with respect to $\mu$ and $\rho_{m}$ through a linear simultaneous equation. This approximation is based on the assumption that $\mu^{(t)} \simeq \mu^{(t-1)}$. In this way, we obtain the update equations given in Section II-B.

\section{ACKNOWLEDGMENT}

The authors would like to thank Dr. J. Le Roux for fruitful discussions and the reviewers for their constructive suggestions that have helped improve the manuscript.

\section{REFERENCES}

[1] F. Itakura and S. Saito, "Analysis synthesis telephony based upon the maximum likelihood method," in Proc. 6th Int. Congr. Acoust. (ICA'68), 1968, C-5-5, C17-20.

[2] B. S. Atal, "Effectiveness of linear prediction characteristics of the speech wave for automatic speaker identification and verification," $J$. Acoust. Soc. Amer., vol. 55, no. 6, pp. 1304-1312, 1974.

[3] M. N. Murthi and B. D. Rao, "All-pole modeling of speech based on the minimum variance distortionless response spectrum," IEEE Trans. Speech Audio Process., vol. 8, no. 3, pp. 221-239, May 2000.

[4] D. Giacobello et al., "Sparse linear predictors for speech processing," in Proc. Interspeech, 2008.

[5] E. Denoël and J.-P. Solvay, "Linear prediction of speech with a least absolute error criterion," IEEE Trans. Acoust., Speech, Signal Process., vol. ASSP-33, no. 6, pp. 1397-1403, Dec. 1985.

[6] A. V. Oppenheim and R. W. Schafer, "Homomorphic analysis of speech," IEEE Trans. Audio Electroacoust., vol. AU-16, no. 2, pp. 221-226, Jun. 1968.

[7] A. El-Jaroudi and J. Makhoul, "Discrete all-pole modeling," IEEE Trans. Signal Process., vol. 39, no. 2, pp. 411-423, Feb. 1991.

[8] R. Badeau and B. David, "Weighted maximum likelihood autoregressive and moving average spectrum modeling," in Proc. Int. Conf. Acoust., Speech, Signal Process. (ICASSP'08), 2008, pp. 3761-3764.

[9] T. Galas and X. Rodet, "An improved cepstral method for deconvolution of source-filter systems with discrete spectra: Application to musical sound signals," in Proc. 1991 Int. Comput. Music Conf. (ICMC'90), 1990, pp. 82-84.

[10] O. Cappé, J. Laroche, and E. Moulines, "Regularized estimation of cepstrum envelope from discrete frequency points," in Proc. IEEE Workshop Appls. Signal Process. Audio Acoust. (WASPAA'95), 1995.

[11] M. Campedel-Oudot, O. Cappé, and E. Moulines, "Estimation of the spectral envelope of voiced sounds using a penalized likelihood approach," IEEE Trans. Speech Audio Process., vol. 9, no. 5, pp. 469-481, Jul. 2001.

[12] H. Kawahara, "Speech respresentation and transformation using adaptive interpolation of weighted spectrum: Vocoder revisted," in Proc. Int. Conf. Acoust., Speech, Signal Process. (ICASSP'97), 1997, vol. 2, pp. 1303-1306.

[13] W. J. Hess, Pitch Determination of Speech Signals. Berlin, Germany: Springer-Verlag, 1983.

[14] W. J. Hess, "Pitch and voicing determination," in Advances in Speech Signal Processing, S. Furui and M. M. Sohndi, Eds. New York: Marcel Dekker, 1992, pp. 3-48.
[15] A. P. Klapuri, "Multiple fundamental frequency estimation based on harmonicity and spectral smoothness," IEEE Trans. Speech Audio Process., vol. 11, no. 6, pp. 804-816, Nov. 2003.

[16] C. Yeh, A. Roebel, and X. Rodet, "Multiple fundamental frequency estimation of polyphonic music signals," in Proc. 2005 IEEE Int. Conf. Acoust., Speech, Signal Process. (ICASSP'05), 2005, vol. 3, pp. 225-228.

[17] T. Virtanen and A. Klapuri, "Separation of harmonic sounds using linear models for the overtone series," in Proc. 2002 IEEE Int. Conf. Acoust., Speech, Signal Process. (ICASSP'02), 2002, vol. 2, pp. $1757-1760$.

[18] F. Bach and M. Jordan, "Discriminative training of hidden markov models for multiple pitch tracking," in Proc. 2005 IEEE Int. Conf. Acoust., Speech, Signal Process. (ICASSP'05), 2005, vol. 5, pp. 489-492.

[19] G. Cauwenberghs, "Monaural separation of independent acoustical components," in Proc. 1999 IEEE Symp. Circuit Syst. (ISCAS'99), 1999, pp. 62-65.

[20] R. J. Leistikow, H. D. Thornburg, J. O. Smith, III, and J. Berger, "Bayesian identification of closely-spaced chords from single-frame STFT peaks," in Proc. 17th Int. Conf. Digital Audio Effects (DAFx04), 2004, pp. 5-8.

[21] A. P. Dempster, N. M. Laird, and D. B. Rubin, "Maximum likelihood from incomplete data via the EM algorithm," J. R. Statist. Soc., ser. B, vol. 39, no. 1, pp. 1-38, 1977.

[22] I. Csiszár, " I-divergence geometry of probability distributions and minimization problems," Ann. of Probability, vol. 3, no. 1, pp. 146-158, 1975.

[23] D. D. Lee and H. S. Seung, "Algorithms for non-negative matrix factorization," in Proc. 2000 Adv. Neural Inf. Process. Syst. (NIPS'00), 2000, pp. 556-562.

[24] K. Lange, D. Hunter, and I. Yang, "Optimization transfer using surrogate objective functions," J. Comp., Graph. Statist., vol. 9, no. 1, pp. $1-20,2000$

[25] H. Kuwabara, K. Takeda, Y. Sagisaka, S. Katagiri, S. Morikawa, and T. Watanabe, "Construction of a large-scale Japanese speech database and its management system," in Proc. 1989 IEEE Int.. Conf. Acoust., Speech, Signal Process. (ICASSP'89), 1989, pp. 560-563.

[26] A. de Cheveigné and H. Kawahara, "YIN, a fundamental frequency estimator for speech and music," J. Acoust. Soc. Amer., vol. 111, no. 4, pp. 1917-1930, 2002.

[27] H. Kameoka, T. Nishimoto, and S. Sagayama, "A multipitch analyzer based on harmonic temporal structured clustering," IEEE Trans. Audio, Speech, Lang. Process., vol. 15, no. 3, pp. 982-994, Mar. 2007.

[28] J. Le Roux, H. Kameoka, N. Ono, A. de Cheveigné, and S. Sagayama, "Single and multiple pitch contour estimation through parametric spectrogram modeling of speech in noisy environments," IEEE Trans. Audio, Speech, Lang. Process., vol. 15, no. 4, pp. 1135-1145, May 2007.

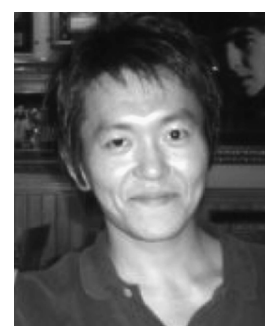

Hirokazu Kameoka (M'07) received B.E., M.E., and $\mathrm{Ph} . \mathrm{D}$. degrees all from the University of Tokyo, Tokyo, Japan, in 2002, 2004, and 2007, respectively. $\mathrm{He}$ is currently a Research Scientist at NTT Communication Science Laboratories, Kanagawa, Japan. His research interests include computational auditory scene analysis, acoustic signal processing, speech analysis, and music applications.

Dr. Kameoka is a member of the Institute of Electronics, Information and Communication Engineers (IEICE), the Information Processing Society of Japan (IPSJ), and the Acoustical Society of Japan (ASJ). He was awarded the Yamashita Memorial Research Award by IPSJ, the Best Student Paper Award Finalist at 2005 IEEE International Conference on Acoustics, Speech, and Signal Processing (ICASSP2005), the 20th Telecom System Technology Student Award by the Telecommunications Advancement Foundation (TAF) in 2005, the Itakura Prize Innovative Young Researcher Award by ASJ, 2007 Dean's Award for Outstanding Student in the Graduate School of Information Science and Technology by the University of Tokyo, 1st IEEE Signal Processing Society Japan Chapter Student Paper Award in 2007, the Awaya Prize Young Researcher Award by ASJ in 2008, and the IEEE Signal Processing Society 2008 SPS Young Author Best Paper Award in 2009. 


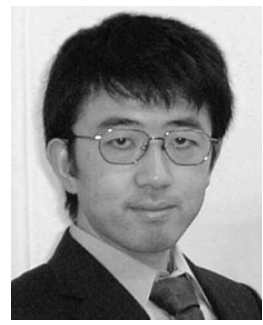

Nobutaka Ono (M'02) received the B.E., M.S., and $\mathrm{Ph} . \mathrm{D}$. degrees in mathematical engineering and information physics from the University of Tokyo, Tokyo, Japan, in 1996, 1998, and 2001, respectively.

He has been with the Graduate School of Information Science and Technology, University of Tokyo, as a Research Associate since 2001, and as a Lecturer since 2005. His research interests include acoustic signal processing, speech processing, music processing, sensing and measurement, and auditory modeling.

Dr. Ono was the Secretary of the Technical Committee of Psychological and Physiological Acoustics in Japan from 2006 to 2009. He is a member of the Institute of Electronics, Information and Communications Engineers (IEICE), the Institute of Electrical Engineers of Japan (IEEJ), the Acoustical Society of Japan (ASJ), the Society of Instrument and Control Engineers (SICE), and the Information Processing Society of Japan (IPSJ). He received the Sato Prize Paper Award from ASJ in 2000, the Igarashi Award at the Sensor Symposium on Sensors, Micromachines, and Applied Systems from IEEJ in 2004, the Awaya Prize Young Researcher Award from ASJ in 2007, and the Best Paper Award at the International Symposium on Industrial Electronics (ISIE) in 2008.

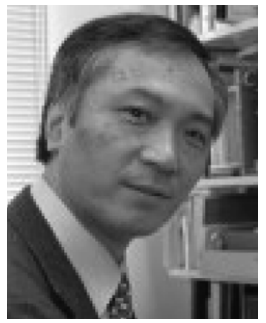

Shigeki Sagayama (M'82) received the B.E., M.E., and $\mathrm{Ph} . \mathrm{D}$. degrees from the University of Tokyo, Tokyo, Japan, in 1972, 1974, and 1998, respectively, all in mathematical engineering and information physics.

He joined Nippon Telegraph and Telephone Public Corporation (currently, NTT) in 1974 and started his career in speech analysis, synthesis, and recognition at NTT Labs in Musashino, Japan. From 1990, he was Head of the Speech Processing Department, ATR Interpreting Telephony Laboratories, Kyoto, Japan where he was in charge of an automatic speech translation project. In 1993, he was responsible for speech recognition, synthesis, and dialog systems at NTT Human Interface Laboratories, Yokosuka, Japan. In 1998, he became a Professor of the Graduate School of Information Science, Japan Advanced Institute of Science and Technology (JAIST), Ishikawa. In 2000, he was appointed Professor at the Graduate School of Information Science and Technology (formerly, Graduate School of Engineering), the University of Tokyo. His major research interests include the processing and recognition of speech, music, acoustic signals, handwriting, and images. He was the leader of anthropomorphic spoken dialog agent project (Galatea Project) from 2000-2003.

Prof. Sagayama received the National Invention Award from the Institute of Invention of Japan in 1991, the Chief Official's Award for Research Achievement from the Science and Technology Agency of Japan in 1996, and other academic awards including Paper Awards from the Institute of Electronics, Information and Communications Engineers, Japan (IEICEJ) in 1996 and from the Information Processing Society of Japan (IPSJ) in 1995. He is a member of the Acoustical Society of Japan, IEICEJ, and IPSJ. 